\title{
Schmale Förderung bei hohen Risiken
}

\section{Arbeitsmarktpolitik für junge Menschen}

\author{
Tina Hofmann
}

Tina Hofmann ist Referentin für Jugendsozialarbeit beim Paritätischen Gesamtverband in Berlin.

E-Mail

jugendsozialarbeit@paritaet.org
Trotz des vielbeschworenen demographischen Wandels und absehbaren Arbeitskräftemangel stehen junge Menschen nicht mehr länger im Blickpunkt der Arbeitsmarktpolitik. Einige Träger der Jugendsozialarbeit haben daraus bereits konzeptionelle Konsequenzen gezogen, indem sie sich neue Kooperationspartner wie Jugendämter, Schulen und Unternehmen suchen.

Junge Menschen standen noch bis vor kurzem im Zentrum der Arbeitsmarktpolitik. Das hat sich mittlerweile verändert. Ist dies durch die Entwicklungen am Arbeits- und Ausbildungsstellenmarkt für junge Menschen begründet?

Erste These: Der offene Arbeitsmarkt hat den Preis eines prekären Einstiegs

Die Jugendarbeitslosigkeit in Deutschland ist im internationalen Vergleich seit vielen Jahren vergleichsweise niedrig. Im Mai 2011 waren 5,5\% der 15- bis 25Jährigen offiziell arbeitslos gemeldet. (1) Auch in Zeiten der Wirtschaftskrise waren junge Menschen nur etwas häufiger arbeitslos als noch vor zehn Jahren. (2) Allerdings erlangen junge Menschen ihre vergleichsweise guten Eintrittschancen am Arbeitsmarkt zu ungünstigen Beschäftigungsbedingungen. Im letzten Jahrzehnt stieg der Anteil junger Erwerbstätiger mit befristeten Stellen. (3) Selbst unter Ausklammerung von Ausbildungsphasen beträgt der Anteil befristet tätiger junger Erwerbstätiger in der Altersgruppe 15 bis 20 Jahren rund $41 \%$. In der Altersgruppe der 20- bis unter 25Jährigen arbeitete gut jeder Vierte mit befristetem Vertrag.

Der Eintritt ins Erwerbsleben ist prekär und verschiebt zeitlich sich ins Erwachsenenalter, weil junge Menschen heute im Vergleich zu früheren Zeiten längere Phasen in Bildung und Ausbildung haben.

\section{Zweite These: Der Übergang von der Schule in den Beruf ist durch ungleiche Bildungs- chancen und Risiken sozialer Ausgrenzung geprägt}

Leider schlägt sich die längere Ausbildungszeit nicht generell in höheren Bildungsabschlüssen und verbesserten Chancen auf einen sozialen Aufstieg nieder: Einerseits nahm die Zahl der Schulabgänger mit Hochschulreife in den letzten Jahren zu, so dass heute knapp jeder dritte Schulabgänger ein Studium aufnehmen kann. (4) Andererseits gibt es nach wie vor zu viele Jugendliche, die die Regelschule ohne Schulabschluss verlassen. In den Jahren 2004 bis 2008 sank die Zahl der Schulabgänger ohne Schulabschluss nur um 1\% auf 7,5\%. (5) Der Anteil von jungen Menschen ohne Berufsabschluss ist in den letzten Jahren sogar gestiegen. Im Jahr 2008 lag der Anteil Ungelernter in der Altersgruppe von 20 bis 29 Jahren bei $15,2 \%$. (6)

Durchgehend schwierig haben es ausländische Jugendliche, einen Berufsabschluss zu erlangen. Ihre UngelerntenQuote liegt sei vielen Jahren unverändert bei rund 30\%. Dieser auch angesichts des demographischen Wandels äußerst negativ zu bewertende Trend hat mit jahrlang verfestigten Problemen am Ausbildungsstellenmarkt zu tun.

Weil seit vielen Jahren Ausbildungsplätze fehlen, ist das sogenannte Übergangssystem bis in das Jahr 2010 hinein stetig angewachsen. Die Ausbildungschancen für Jugendliche mit nur Hauptschulabschluss oder Migrationshintergrund sind weiterhin gering. Während sich im letzten Jahr $94 \%$ aller Hauptschüler eine betriebliche Ausbildung wünschten, gelang nur rund einem Drittel (36 \%) tatsächlich der Einstieg in eine Berufsausbildung.

Der Paritätische Gesamtverband hat das Institut für Sozialpädagogische Forschung mit der Untersuchung der Frage betraut, wie groß der Personenkreis der- 
jenigen jungen Menschen einzuschätzen ist, deren Integration ins Erwerbsleben und Gesellschaft absehbar gefährdet ist, etwa weil sei nach Scheitern in Maßnahmen des Übergangssystems nicht in Ausbildung und Arbeit integriert werden können oder aufgrund vielfältiger persönlicher Belastungen der Unterstützung der Jugendhilfe bedürfen. Nach Berechnungen gehören zu dem Personenkreis dieser sogenannten »integrationsgefährdeten Jugendlichen « derzeit rund 537.000 Jugendliche und junge Erwachsene. (7) Wie hat die Arbeitsmarktpolitik der letzten Jahre auf diese herausfordernden Entwicklungen reagiert?

\section{Dritte These: Die Besonderheit der Jugend ist für die Arbeitsmarktpolitik vorbei}

Während die Arbeitsmarkpolitik der Großen Koalition noch von einem besonderen Schwerpunkt zur Bekämpfung der Jugendarbeitslosigkeit bestimmt war, ist kein besonderer Fokus der aktuellen Arbeitsmarktpolitik auf junge Menschen mehr gegeben. Allerdings war der frühere Förderschwerpunkt gerade in der Grundsicherung für Arbeitssuchende mit einem massenhaften, teils zu wenig individuellen Instrumenteneinsatz für junge Menschen verbunden. Gerade mit Einführung der Grundsicherung für Arbeitssuchende landete ein Großteil junger Menschen lediglich in den Ein-Euro-Jobs. In der aktuellen Ausrichtung der Arbeitsmarktpolitik sind nur wenige zielgruppenspezifische Schwerpunkte, diese für ältere Menschen und zugunsten von Menschen mit Behinderungen, erkennbar.

\section{Vierte These: Es gibt neue}

Akzente bei den präventiven Hilfen in Schulen, aber Maßnahmen zum Nachholen von Schulabschlüssen sind fast ohne Bedeutung

Mit den jüngsten arbeitsmarktpolitischen Reformen wurde den Arbeitsagenturen ein neues Förderinstrument zur präventiven Hilfe an die Hand gegeben, welches zusätzlich durch das Programm »Bildungsketten « des Bildungsministeriums befördert wurde. Die sogenannte Be- rufseinstiegsbegleitung setzt mit der Förderung schon in der Schule an und hilft Jugendlichen, sowohl einen Schulabschluss zu erlangen als auch den Weg in die Berufsausbildung zu finden.

Geht es nach den Vorschlägen der Bundesregierung zur aktuellen Reform der arbeitsmarktpolitischen Instrumente (8), so wird das zunächst nur befristete Instrument fortgeführt. Zukünftig sollen die Arbeitsagenturen die Förderung aber nur noch unter der Voraussetzung leisten, dass sich weitere Finanzierungspartner mit mindestens $50 \%$ an den Kosten beteiligen.

Verstetigt und ausgebaut werden könnte die Förderung der Berufsorientierung an Schulen. Das Angebot soll außerdem verstärkt für Schülerinnen und Schüler mit Behinderungen zugänglich gemacht werden, die als Abgänger von Förderschulen heute zu wenig Chancen auf dem allgemeinen Arbeitsmarkt haben und häufig in Werkstätten für Menschen mit Behinderungen gelangen.

Die präventiven Förderinstrumente der Berufsorientierung und Berufseinstiegsbegleitung haben in den letzten Jahren an Bedeutung gewonnen; so wurden im Mai 2011 rund 75.000 Jugendliche gefördert. (9)

Mehr Symbolwirkung als praktische Bedeutung hat hingegen der Rechtsanspruch in der Arbeitsförderung auf das Nachholen eines Schulabschlusses für junge Menschen erlangt. Die Große Koalition hatte gezielt Rechtsansprüche in der Arbeitsförderung verankert, damit Jugendliche und junge Erwachsene in Berufsvorbereitenden Bildungsmaßnahmen nachträglich einen Schulabschluss erwerben können. Hiervon konnten im Jahr 2009 lediglich rund 6.000 Jugendliche profitieren. (10)

\section{Fünfte These: Der Rückzug bei der Ausbildungsförderung ist eingeläutet}

Ungeachtet der andauernden Probleme am Ausbildungsstellenmarkt - fehlende Ausbildungsplätze, schlechte Ausbildungschancen für benachteiligte Jugendliche und Migranten, weiterhin hohe Altbewerberzahlen - ist die Ausbildungsförderung im Rückzug begriffen.

Konkret zeigt sich das bereits beispielsweise an der Reduzierung von Ausbil- dungsangeboten, die von der Bundesagentur für Arbeit gefördert werden. Seit Jahresbeginn 2011 sinkt die Zahl der Jugendlichen massiv, die in eine außerbetriebliche Ausbildung einmünden. Im Vorjahresvergleich gelangten rund 30\% weniger junge Menschen in eine solche Ausbildung. (11)

Die Bundesregierung hat sich außerdem von speziellen Förderangeboten für sogenannte Altbewerber verabschiedet, die in den letzen Jahren zu einer großen "Problemgruppe « am Ausbildungsstellenmarkt herangewachsen waren. Sie hat den Ausbildungsbonus mit Verweis auf mögliche Mitnahmeeffekte abgeschafft, ohne dass alternative Förderungen für diese Zielgruppe angeboten worden sind. Derzeit zählen immer noch rund 256.000 Bewerberinnen und Bewerber zu dieser Gruppe. (12)

Der neuerliche Versuch aus dem Arbeitsministerium, sich im Rahmen der aktuellen Reform der arbeitsmarktpolitischen Instrumente auch von der sogenannten Einstiegsqualifizierung zu verabschieden, wurde durch breiten Protest von Wirtschaft, Jugend- und Sozialverbänden unterbunden.

\section{Sechste These: Die Kürzungen in der Arbeitsmarktpolitik gehen auch zulasten der Qualifizierung junger Menschen}

Die Förderrückgänge hängen nicht unwesentlich mit den massiven Kürzungen der Mittel für die aktive Arbeitsmarktpolitik zusammen. Die Sparbeschlüsse der Bundesregierung sehen Kürzungen in der aktiven Arbeitsmarktpolitik im Zeitraum 2011 bis 2014 in Höhe von insgesamt 16 Milliarden Euro vor.

Die aktive Arbeitsmarktpolitik und Ausbildungsförderung wird infolgedessen stark eingeschränkt. Nicht nur die Ausbildungsförderung, auch die Fort- und Weiterbildung von jungen Menschen ist rückläufig. Ein Minus von 34\% in der Weiterbildung junger Menschen weist die Förderstatistik der Bundesagentur für Arbeit gegenüber dem Vorjahr aus.

Gezielte Investitionen in die nachträgliche Qualifizierung von jungen Menschen bleiben so auf der Strecke; dies trotz des gestiegenen Anteils ungelernter junger Er- 
wachsener und des absehbaren Fachkräftemangels.

\section{Siebte These: Die soziale Ausgrenzung junger Menschen wird nicht als Thema der Arbeitsmarktpolitik verstanden}

Die aktuelle Arbeitsmarktpolitik macht es sich nicht zur Aufgabe, der sozialen Ausgrenzung von jungen Menschen, die sich beispielsweise an schlechten Wohnverhältnissen, schwierigen familiären Bedingungen, dauerhaft verwehrten Zugängen zu Bildung und Qualifizierung zeigt, entgegenzuwirken. Zwar wurde mit den sogenannten Maßnahmen zur Aktivierung und beruflichen Eingliederung in 2009 ein neues Angebot zur nieder schwelligen Förderung von förderbedürftigen Jugendlichen geschaffen, das von vielen Trägern der Jugendsozialarbeit in ihrer Praxis für eine bedarfsgerechte und auch ausreichend lange Förderung genutzt werden kann. Allerdings beklagen viele Praktiker fehlende Flexibilität in der Förderung und Nachteile, die mit der öffentlichen Ausschreibung dieser Angebote einhergehen. (13) Zudem bestehen in der Grundsicherung für Arbeitsuchende nach wie vor rigide Sanktionsmechanismen, wodurch der sozialen Ausgrenzung von Jugendlichen Vorschub geleistet wird.

Die Kooperation der Arbeitsförderung mit anderen Hilfesystemen, die in der Lage wären, komplexe Problemlagen aus dem Lebensumfeld junger Menschen aufzugreifen, ist noch unterentwickelt. Das gilt vor allem für die Zusammenarbeit von Jugendämtern mit Jobcentern und Arbeitsagenturen.

Bessere Voraussetzungen für die Kooperation müssten auch bei der Gestaltung arbeitsmarktpolitischer Instrumente geschaffen werden, etwa durch geeignete Finanzierungsregelungen. Dies war allerdings weder Gegenstand der letzten großen Reform der arbeitsmarktpolitischen Instrumente noch ist es Thema der aktuellen Gesetzgebung. Offenbar geht es derzeit vor allem darum, feste Zuständigkeitsgrenzen für die Arbeitsmarktpolitik zu wahren und damit Steuerung und Kostenkontrolle zu behalten.

\section{Ausblick}

Jugendliche und junge Erwachsene stehen nicht länger im Fokus der Arbeitsmarktpolitik. So sehr sich die Förderung der Arbeitsagentur auch mit neueren Förderinitiativen zuletzt an den präventiven Bereich der Schulen verlagert hat, so vorschnell zieht sie sich aus dem Bereich der Ausbildungsförderung zurück. Dies geschieht zu einem Zeitpunkt, da der demographische Wandel allenfalls regionale Entlastungen am Ausbildungsstellenmarkt gebracht hat. Im Zuge der aktuellen drastischen Kürzungen in der Arbeitsmarktförderung fehlen notwendige Mittel, um in die Qualifikationen vieler ungelernter junger Erwachsener zu investieren und damit einen Beitrag zur Bekämpfung des Fachkräftemangels zu leisten.

Mit der wachsenden sozialen Ausgrenzung junger Menschen werden sich zukünftig immer stärker Kommunen und die kommunale Jugendhilfe konfrontiert sehen. Die Träger der Jugendsozialarbeit haben sich auf den Weg gemacht, die aktuellen Entwicklungen der Arbeitsmarktpolitik zu bewältigen. Sie sind nicht mehr alleine in der beruflichen Förderung junger Menschen im Auftrag von Jobcentern und Arbeitsagenturen tätig, sondern haben sich weitere Standbeine, etwa an Schulen, in der Zusammenarbeit mit Unternehmen oder bei der Förderung von Familien geschaffen. Damit gelingt ihnen ein notwendiger Blick auf junge Menschen, den die aktuell von Kürzungen geprägte Arbeitsmarktförderung gar nicht einnehmen kann: die Sichtweise auf den ganzen Menschen und seine Lebensumstände.

\section{Anmerkungen}

(1) Bundesagentur für Arbeit (Hg.): Der Arbeits- und Ausbildungsmarkt in Deutschland. Mai 2011.

(2) Aktuelle Meldungen des Statistischen Bundesamts zum Arbeitsmarkt vom 24.6.2011 veröffentlicht unter www.destatis.de.

(3) Statistisches Bundesamt a. a. O.

(4) Autorengruppe Bildungsberichterstattung im Auftrag der Ständigen Konfe- renz der Kultusminister der Länder in der Bundesrepublik Deutschland und des Bundesministeriums für Bildung und Forschung (Hg.) 2010: Bildung in Deutschland 2010. Ein indikatorengestützter Bericht mit einer Analyse zu Perspektiven des Bildungswesens im demografischen Wandel. Ein indikatorengestützter Bericht mit einer Analyse zu Perspektiven des Bildungswesens im demografischen Wandel. Bielefeld.

(5) Siehe Autorengruppe Bildungsberichterstattung a. a. O.

(6) DRS 17/5344.

(7) Institut für Sozialpädagogische Forschung (2011): Expertise zum Positionspapier »Ausgrenzungsprozessen entgegentreten -Neujustierung der Hilfen für Jugendliche und junge Erwachsene. « Mainz.

(8) Siehe Entwurf eines Gesetzes zur Leistungssteigerung am Arbeitsmarkt.

(9) Angaben aus BA-Förderstatistik.

(10) Für Jugendliche ist das Nachholen des Hauptschulabschlusses innerhalb einer Berufsvorbereitenden Bildungsmaßnahme möglich. Im Jahr 2009 haben $5 \%$ der Teilnehmenden einer solchen Maßnahme einen Hauptschulabschluss nachträglich erwerben können, dies waren rund 6.000 Jugendliche, siehe INBAS (Hg.) 2010: Erfolgreich bestanden. Parameter für den nachträglichen Erwerb des Hauptschulabschlusses. Gutachten zur Ausgestaltung von vorbereitenden Kursen zum nachträglichen Erwerb des Hauptschulabschlusses im Auftrag des Bundesministeriums für Arbeit und Soziales. Offenbach am Main.

(11) Kooperationsverbund Jugendsozialarbeit (2011): Ausbildung (wieder nicht) für alle?. Stellungnahme des Kooperationsverbundes Jugendsozialarbeit zum Berufsbildungsbericht 2011. Berlin.

(12) Bundesinstitut für Berufsbildung (Hg.) 2011: Datenreport zum Berufsbildungsbericht 2011. Bonn.

(13) Institut für Angewandte Wirtschaftsforschung e. V. (2011): Expertise zur Umsetzung ausgewählter Instrumente des Gesetzes zur Neuausrichtung der arbeitsmarktpolitischen Instrumente für benachteiligte Jugendliche und junge Erwachsene (U25). Tübingen. 